\title{
Evaluation de la prévalence de l'anémie chez les élèves bénéficiaires du programme d'alimentation scolaire du PAM (2015-2017) à Nara et à Kolokani au Mali
}

\section{Evaluation of the prevalence of anemia among students benefiting from the WFP feeding program (2015- 2017) in Nara and Kolokani in Mali}

\author{
TOURE ${ }^{1}$ O., TOURE ${ }^{1}$ H., LANDOURE ${ }^{1}$ A., SANGHO² O., COULIBALY² CA., SANGHO² A., TOGO ${ }^{2}$ Y. TELLY² N.
}

\author{
1 Institut National de Santé Publique, \\ 2 Département d'Enseignement et de Recherche en \\ Santé Publique \\ ${ }^{3}$ Faculté de Pharmacie, USTTB
}

\section{Auteur correspondant : oussou_toure@hotmail.com Résumé}

La malnutrition chez les enfants de moins de cinq ans, peut contribue à la diminution de la capacité intellectuelle; il a été aussi accepté que l'insécurité alimentaire, cause de la malnutrition dont un des indicateurs est l'anémie, est une cause d'absentéisme et de baisse de la capacité d'apprentissage chez les enfants d'âge scolaire. La Politique du Mali de promotion de Cantines Scolaires s'est compte sur l'engagement du PAM à couvrir les besoins alimentaires des cantines des écoles fondamentales des cercles de Nara et de Kolokani. L'objectif de cette recherche était d'évaluer le niveau de la prévalence de l'anémie chez les élèves des écoles bénéficiaires du soutien du PAM, afin de disposer d'indicateurs de base permettant à cette organisation d'évaluer l'impact de ses interventions. Sur la base de la liste et des effectifs des écoles, on a procédé à un tirage au hasard d'un échantillon de 27 écoles, puis, proportionnellement à l'effectif de chaque école, 20 à 30 élèves ont été retenus; soit un total de 560 élèves. Le taux d'hémoglobine chez chaque élève a été déterminé sur place avec l'hémoglobinomètre portatif « HemoCue ». Au total, 170 élèves sur les 560 enrôlés dans les deux cercles, présentaient une anémie avec une prévalence globale de $35,36 \%$ (29.1 \% à Kolokani et $32.1 \%$ à Nara). Des interventions préventives et correctrices urgentes ont été formulées pour l'administration scolaire et ses partenaires.

Mots clés : Anémie, élèves, programme d'alimentation scolaire, Nara et Kolokani/Mali

\begin{abstract}
Malnutrition in children under the age of five can contribute to the reduction of intellectual capacity; it was also accepted that food insecurity, the cause of malnutrition, one of the indicators of which is anemia, is a cause of absenteeism and reduced learning capacity among school-aged children. Mali's policy for the promotion of school canteens is based on WFP's commitment to cover the food needs of the canteens of the basic schools of the circles of Nara and Kolokani. The objective of this research was to assess the level of anemia prevalence among students in schools receiving
\end{abstract}

WFP support, in order to have basic indicators allowing this organization to assess the impact of its interventions. On the basis of the list and the number of schools, a sample of 27 schools was drawn at random, then, in proportion to the number of each school, 20 to 30 pupils were selected; a total of 560 students. The hemoglobin level in each student was determined on site with the portable Hemoglobinometer "HemoCue". A total of 170 pupils out of the 560 enrolled in the two circles presented anemia with an overall prevalence of $35.36 \%(29.1 \%$ in Kolokani and $32.1 \%$ in Nara). Urgent preventive and corrective interventions have been formulated for the school administration and its partners.

Keys Words: Anemia, students, school feeding program, Nara and Kolokani / Mali

\section{INTRODUCTION}

L'anémie par déficience en fer, ou anémie ferriprive, est le type d'anémie que l'on rencontre le plus souvent dans tous les groupes d'âge (OMS / UNICEF, 2004).

L'insuffisance de fer est la forme la plus commune de malnutrition dans le monde; et selon l'Organisation mondiale de la Santé, $43 \%$ des enfants du monde souffrent d'anémie (De Maeyer et Adiels-Teasman, 1985).

L'insuffisance peut être due à l'ingestion diététique du fer, le niveau bas de l'absorption en raison de la petite pathologie d'entrailles qui rend les conditions physiologiques accrues de la croissance pendant l'enfance et l'adolescence, la perte chronique de sang habituellement des appareils urinaires et gastrointestinaux ou en raison de l'hémorragie chez les filles adolescentes. Cependant l'anémie est due le plus souvent à un apport insuffisant de fer dans l'alimentation. Le Programme Alimentaire Mondial (PAM) a démarré en janvier 2015 un nouveau programme d'assistance au Mali (2015-2017). Une des composantes de ce programme concerne l'alimentation scolaire des élèves fréquentant l'enseignement élémentaire du premier cycle dans les zones à insécurité alimentaire des régions de Gao, Tombouctou, Mopti, Koulikoro et Kayes.

L'assistance consiste à la fourniture des repas chauds aux élèves à la mi-journée pendant toute l'année scolaire (180 jours/an). Le panier alimentaire est composé de céréales, légumineuses, huile végétale, sel iodé et poudre en micronutriments (MNP). Quoique faisant partie du panier alimentaire, le MNP n'est pas encore disponible à cause des contraintes financières du moment. 
Dans la région de Koulikoro, le PAM couvre 54 écoles: 24 dans le cercle de Nara (effectif de 3661 élèves), et 30 dans le cercle de Kolokani (effectif de 6108 élèves); soit un total 9.769 élèves dans les deux cercles.

Avant l'introduction du MNP comme repas journaliers des enfants, le PAM a souhaité disposer des données de base de référence, à travers une étude, qui aideront, à mieux mesurer l'effet ou l'impact du MNP sur l'état nutritionnel des écoliers tout au long de la période d'assistance. C'est dans ce cadre que le PAM a confié à I'INRSP la présente étude d'évaluation du statut nutritionnel des enfants basée sur un test de détermination du taux d'hémoglobine chez l'individu comme indicateur de la prévalence de l'anémie.

\section{METHODOLOGIE}

\section{Echantillonnage}

Sur base de la liste des écoles et des effectifs assistés, on a procédé à un tirage au hasard d'un échantillon d'écoles, puis, proportionnellement à l'effectif de chaque école, on a déterminé le nombre d'élèves à enquêter, soit 20 à 30 élèves par école. L'échantillon a été choisi de sorte qu'il soit représentatif et par conséquent les résultats obtenus sont applicables à la population étudiée.

Les écoles ont été tirées suivant la méthode échantillonnage systématique ; ainsi une école sur deux a été tirée (voir liste des écoles retenues). Sur cette base, on a sélectionné 12 écoles à Nara, et 15 écoles à Kolokani. Dans chaque école, 20 ou 30 élèves ont été tirés au hasard et testés. A Kolokani, ont été retenues 20 élèves (10 filles et 10 garçons) dans chacune des 13 écoles et 30 élèves dans les deux autres écoles restantes, à cause de leurs effectifs plus importants. Au total, 560 élèves ont subi le test dans les deux cercles.

Détermination du taux d'hémoglobine

Pour tester l'anémie, il a été effectué un prélèvement d'une goutte de sang au niveau du doigt et grâce à l'Hémoglobinomètre portatif "HemoCue », le taux d'hémoglobine a été déterminé sur place.

\section{Analyse et interprétation des résultats}

Les données collectées ont été vérifiées pour un nettoyage qualitatif, avant leur saisie. Elles ont été ensuite saisies sur le logiciel Excel Windows 2007 et analysées par le logiciel SPSS .PC 21. La classification des écoliers en statut anémié ou non a été faite selon les critères de l'OMS, comme suit : L'anémie est sévère si le taux d'hémoglobine est inférieur à $7,0 \mathrm{~g} / \mathrm{dl}$, Elle est modérée si le taux se situe entre $7,0 \mathrm{~g} / \mathrm{dl}$ et $9,9 \mathrm{~g} / \mathrm{dl}$, et L'anémie est légère si le taux se situe entre $10,0 \mathrm{~g} / \mathrm{dl}$ et $10,9 \mathrm{~g} / \mathrm{dl}$. Ainsi tout enfant de 6 à 16 ans dont le taux d'hémoglobine inférieur à $11,5 \mathrm{~g} / \mathrm{dl}$ est classé anémié.

Considérations d'ordre éthique

Le protocole de l'étude a obtenu le quitus du Comité d'Ethique de l'INRSP avant sa mise en œuvre. Une lettre de consentement éclairé a été préparée et soumise à la lecture et à la signature de chaque Directeur d'école ou son représentant.

\section{RESULTATS}

\section{Caractéristiques socio démographiques}

L'enquête a concerné au total 560 élèves dont 320 (57.1 $\%$ ) à Kolokani et $240(42.9 \%)$ à Nara. Environ le même nombre de filles et de garçons a été enregistré dans les 2 cercles ; l'âge variait de 5 à 16 ans avec un âge moyen de $10.1 \pm 2.6$ à Kolokani contre une variation d'âge et une moyenne, respectivement, de 5 à 15 ans et de $9.3 \pm$ 2.0 à Nara. Le pic de participation était de 60 élèves en faveur des âgés de 12 ans à Kolokani contre 45 élèves en faveur des âgés de 8 ans à Nara.

Anémie globale chez les écoliers

Le taux d'hémoglobine moyen est d'environ $11,5 \pm 1.2$ $\mathrm{g} / \mathrm{dl}$ avec un minimum de $7.8 \mathrm{~g} / \mathrm{dl}$ et un maximum de 14,7 $\mathrm{g} / \mathrm{dl}$ à Kolokani contre un taux moyen, un minimum et un maximum, respectivement $11,5 \pm 1.4 \mathrm{~g} / \mathrm{dl}, 3,3 \mathrm{~g} / \mathrm{dl}$ et 15.1 $\mathrm{g} / \mathrm{dl}$ à Nara. Les taux les plus faibles correspondant à une anémie sévère ont été enregistrés à Nara chez un garçon de 9 ans $(3,3 \mathrm{~g} / \mathrm{dl})$ et une fille de 12 ans $(6.0 \mathrm{~g} / \mathrm{dl})$. Toujours dans ce cercle 18 écoliers sont atteints de malnutrition modérée, soit $7,5 \%$ des écoliers des écoles couvertes et $23,4 \%$ des enfants anémiés. II n'a pas été enregistré d'anémie sévère chez les écoliers du cercle de Kolokani. Dans ce cercle 31 écoliers souffrent d'anémie modérée soit $9,7 \%$ des écoliers des écoles couvertes et $33,3 \%$ des enfants anémiés.

\section{Prévalence de l'anémie par sexe}

L'anémie à Kolokani a touché au total 93 écoliers soit une prévalence de $(29.1 \%)$ repartie entre 46 garçons $(49.5 \%$ ) et 47 filles (50.5\%) (Tableau I), et un total de 77 écoliers à Nara soit une prévalence de $(32.1 \%)$ repartie entre 52 garçons (67.5\%) et 25 filles (32.5\%) (Tableau I). Apparemment les garçons et les filles sont touchés à des niveaux semblables à Kolokani, par contre, les garçons semblent plus touchés à Nara.

Mais dans les deux cercles, l'anémie modérée semble toucher plus les garçons que les filles ; en effet il a été enregistré 13 garçons anémiés modérés contre 5 filles à Nara, et 18 garçons contre 13 filles à Kolokani.

\section{Prévalence de l'anémie par âge}

La répartition de l'anémie par âge montre une prévalence variant de $0-41.7 \%$, avec un pic de $41.7 \%$ chez les élèves âgés de 7ans à Kolokani (tableau II). A Nara elle variait de 0-50\% avec un pic de $50 \%$ aussi chez les 7 ans (tableau III). Excepté les enfants âgés de 16 ans à Kolokani et ceux âgés de 5 et 15 ans à Nara, tous les autres groupes d'âges étaient atteints de l'anémie à des niveaux variables. Le faible effectif de leur participation à l'enquête pourrait expliquer la non anémie chez les groupes d'âge de 16 ans à Kolokani et 5 à 15 ans à Nara.

Prévalence de l'anémie par école dans le cercle de Kolokani

La répartition de l'anémie par école dans le cercle de Kolokani montre une variation de la prévalence de 15- 
$60 \%$ dans les écoles; toutes les écoles étaient touchées à des degrés différents, et le pic de la prévalence de $60 \%$ est noté à l'école de Kenekolo dans la commune de Tioribougou (Tableau IV). II n'y avait aucun cas d'anémie sévère parmi les écoliers de ce cercle.

Prévalence de l'anémie par école dans le cercle de Nara

La répartition de l'anémie par école dans le cercle de Nara a montré aussi une variation de la prévalence de 10- $50 \%$ dans les écoles; toutes les écoles étaient touchées à des degrés différents, et le pic de la prévalence de $50 \%$ est noté à l'école de Kolly dans la commune de Ouagadou (Tableau V). Deux enfants anémiés sévères, une fille âgée de 12 ans (Taux $\mathrm{Hb}=6 \mathrm{~g} / \mathrm{dl}$ ) et un garçon de 9 ans (Taux $\mathrm{Hb}=3,3 \mathrm{~g} / \mathrm{dl}$ ) ont été vus dans ce cercle.

\section{DISCUSSION}

Au regard des résultats de la présente étude, 170 élèves sur les 560 enrôlés dans les deux cercles présentaient une anémie, soit une prévalence globale de $35,36 \%$, dont 93 élèves à Kolokani, avec une prévalence d'environ $29,1 \%$ et 77 élèves à Nara, avec une prévalence de $32,1 \%$. A Kolokani, presque le même nombre de filles et de garçons étaient anémiés. A Nara on note une différence de prévalence entre les garçons et les filles; les premiers semblent être plus touchés que les secondes. Ces résultats sont inférieurs au taux moyen mondial de $43 \%$ observé chez les enfants (De Maeyer et Adiels-Teasman, 1985). L'anémie par déficience en fer, ou anémie ferriprive, est le type d'anémie que l'on rencontre le plus souvent dans tous les groupes d'âge (OMS / UNICEF, 2004).

Dans les deux cercles le pic de l'anémie est enregistré chez les élèves âgés de 7 ans, environ $47 \%$ à Nara et $50 \%$ à Kolokani ; cet âge de recrutement officiel en première année scolaire formelle est aussi le groupe le plus vulnérable (EDS-M V 2012- 2013). Excepté les âgés de 16 ans à Nara et les 5 et 15 ans à Kolokani, tous les autres groupes d'âges étaient atteints de l'anémie à des niveaux variables. Le faible effectif de leur participation à l'enquête pourrait expliquer la non anémie chez les groupes d'âge sus cités ( 5,15 et 16 ans). Une anémie sévère a été détectée chez une fille et un garçon, tous de Nara.

Les résultats mettent en exergue toute la pertinence du choix du PAM; en effet, ces deux cercles étaient en insécurité alimentaire (EFSA 2013, ENSAN 2014). Les taux de prévalence de l'anémie, parfois, au-dessus du seuil critique de $40 \%$ (OMS, 2010b) dans les deux cercles confirment cette situation. Une intervention était donc bien justifiée.

\section{CONCLUSION}

Les résultats mettent en exergue toute la pertinence du choix du PAM ; en effet, ces deux cercles étaient en insécurité alimentaire. Les taux de prévalence de l'anémie, parfois, au-dessus du seuil critique de $40 \%$ (OMS, 2010b) dans les deux cercles confirment cette situation. Une intervention était donc bien justifiée.

\section{REFERENCES}

1. OMS / UNICEF Joint statement: Focusing on anemia, towards an integrated approach for effective anemia control. OMS, 2004.

2. De Maeyer EM. Adiels-Tegman M., 1985, the prevalence of anemia in the world. Rapp Trimest Stat Sanit Mond, 38: 302-16.

3. World Health Organization/United Nations University/UNICEF., 2001 Iron Deficiency Anemia, Assessment, Prevention and Control: a Guide for Program Managers. WHO, Geneva, Switzerland

4. Stratégie WASH-NUT 2012- 2015 de l'Afrique de l'Ouest et du Centre

Pelletier DI, Bulletin of the World Health Organization. 1995, 73 (4): 443:448

5. INSTAT. Enquête par Grappe à Indicateurs Multiples (MICS) ; République du Mali : 2009.

6. INSTAT. Enquête Nationale Nutritionnelle Anthropométrique et de Mortalité rétrospective, Mali 2011.

7. UNICEF. Technique Simplifiée de sondage pat lots appliques a l'assurance qualité pour évaluer l'accès et la couverture (SLEAC) Mali 2014, CMN

8. Profil nutritionnel du Mali (FAO, 2010)

9. Enquête Nationale sur la Sécurité Alimentaire et Nutritionnelle (ENSAN Mali 2014)

10. Integrated Rural Program to improve Nutrition and Hygiene in Mali (USAID 2014)

11. Enquête Nutritionnelle Anthropométrique et de Mortalité rétrospective (SMART 2015, rapport provisoire)

12. Enquêtes de Démographie et de Santé au Mali (EDSM I à V)

13. http://www.coverage-monitoring.org/usefulreports/mali/

Liste des tableaux :

Tableau II : Prévalence de l'anémie par âge dans les écoles de Kolokani

\begin{tabular}{lcccc}
\hline & \multicolumn{2}{c}{ Anémie } & Non Anémie & \\
\cline { 2 - 4 } Age & $\%$ & $\mathbf{n}$ & $\mathbf{n}$ & Total \\
\hline 5 & 33,3 & 2 & 4 & 6 \\
6 & 35,0 & 7 & 13 & 20 \\
7 & 41,7 & 15 & 21 & 36 \\
8 & 33,3 & 15 & 30 & 45 \\
9 & 38,0 & 11 & 18 & 29 \\
10 & 18,6 & 8 & 35 & 43 \\
11 & 21,0 & 4 & 15 & 19 \\
12 & 26,7 & 16 & 44 & 60 \\
13 & 26,7 & 8 & 22 & 30 \\
14 & 19,0 & 4 & 17 & 21 \\
\hline
\end{tabular}


MALI SANTE PUBLIQUE 2019 SOMASAP

\begin{tabular}{lcccc}
\hline 15 & 30,0 & 3 & 7 & 10 \\
16 & 0,0 & 0 & 1 & 1 \\
Total & $\mathbf{2 9 , 1}$ & $\mathbf{9 3}$ & $\mathbf{1 6 3}$ & $\mathbf{3 2 0}$ \\
\hline
\end{tabular}

Tableau III : Prévalence de l'anémie par âge dans les écoles de Nara

\begin{tabular}{ccccc}
\hline \multirow{2}{*}{ Age } & \multicolumn{2}{c}{ anémie } & Non anémie & Total \\
\cline { 2 - 4 } & $\%$ & $\mathbf{n}$ & $\mathbf{n}$ & \\
\hline 5 & 0,0 & 0 & 2 & 2 \\
6 & 17,6 & 3 & 14 & 17 \\
7 & 50,0 & 17 & 17 & 34 \\
\hline
\end{tabular}

\begin{tabular}{lcccc}
\hline 8 & 42,2 & 19 & 26 & 45 \\
9 & 35,1 & 13 & 24 & 37 \\
10 & 25,0 & 8 & 24 & 32 \\
11 & 24,3 & 9 & 28 & 37 \\
12 & 25,0 & 6 & 18 & 24 \\
13 & 0,0 & 0 & 4 & 4 \\
14 & 28,6 & 2 & 5 & 7 \\
15 & 0,0 & 0 & 1 & 1 \\
Total & $\mathbf{3 2 , 1}$ & $\mathbf{7 7}$ & $\mathbf{1 6 3}$ & $\mathbf{2 4 0}$ \\
\hline
\end{tabular}

Tableau I : Prévalence de l'anémie par sexe à Kolokani et Nara

\begin{tabular}{lllcc}
\hline Cercle & Sexe & Anémie & Non anémie & Total \\
\hline KOLOKANI & Total & $93(29,1 \%)$ & 227 & 320 \\
& Masculin & $46(49,5 \%)$ & 113 & 159 \\
& Féminin & $47(50,5 \%)$ & 114 & 161 \\
NARA & & & & \\
& Total & $77(32,1 \%)$ & 163 & 240 \\
& Masculin & $52(67,5 \%)$ & 77 & 129 \\
& Féminin & $25(32,5 \%)$ & 96 & 111 \\
\hline
\end{tabular}

Tableau IV : Prévalence de l'anémie par école et par commune dans le cercle de Kolokani

\begin{tabular}{llcc}
\hline Commune & Ecoles enquêtées & Prévalence anémie \\
\hline \multirow{3}{*}{ Massantola } & N'Tjinifiana & $\mathrm{N}$ & $\%(\mathrm{n})$ \\
& Kognoumani & 20 & $20,0(4)$ \\
& Massantola & 20 & $30.0(6)$ \\
& Diamanicoura & 20 & $35,0(7)$ \\
Tioribougou & Kenekolo & 20 & $35,0(7)$ \\
Nonssombougou & Kodian & 20 & $60,0(12)$ \\
Ouolodo & N'Tjilla A & 20 & $30,0(6)$ \\
& & 20 & $25,0(5)$ \\
& Djekouma & & \\
Nonkon & Kena & 30 & $26,7(8)$ \\
& Bougoudiana & 20 & $20,0(4)$ \\
Guihoyo & Fondobougou & 30 & $23,3(7)$ \\
& Flabougou & 20 & $20,0(4)$ \\
& Djinidiabougou & 20 & $30,0(6)$ \\
Diedieni & Balla & $25,0(5)$ \\
Sébécoto I & Sanankoroni & 20 & $15,0(3)$ \\
Total & & 320 & $45,0(9)$ \\
\hline
\end{tabular}


Tableau V : Prévalence de l'anémie par école et par commune dans le cercle de Nara

\begin{tabular}{llcc}
\hline Commune & Ecole enquêtées & N & Prévalence anémie \\
& & & $\%(\mathrm{n})$ \\
Dogofry & Kassékaré & 20 & $10,0(2)$ \\
& Gassanbaro & 20 & $35,0(7)$ \\
Dilly & Allasso & 20 & $30,0(6)$ \\
& Dally & 20 & $30,0(6)$ \\
Fallou & Karfabougou & 20 & $20,0(4)$ \\
Koronga & Moussawély & 20 & $40,0(8)$ \\
Nara & Keibane Soninké & 20 & $35,0(7)$ \\
Fallou & Djemene & 20 & $45,0(9)$ \\
Dogofry & Sampaga & 20 & $45,0(9)$ \\
Ouagadou & Kolly & 20 & $50,0(10)$ \\
Koronga & Missira & 20 & $35,0(7)$ \\
Nara & De daye & 20 & $10,0(2)$ \\
Total & & 240 & $32,1(77)$ \\
\hline
\end{tabular}

